communities to educate people about the causes and prevention of diarrhoea. ${ }^{5}$ It has proved difficult to convince people to add bleach to drinking water because it affects the taste. ${ }^{2}$ Moreover, bleach may not be effective in water that is turbid or contains chlorine resistant organisms such as Cyclospora cayetanensis or Cryptosporidium parvum. ${ }^{6}$

Until recently, interventions to improve the safety of water and sanitation have focused on safe disposal of excreta and proper use of water for personal hygiene rather than on paying attention to water quality. ${ }^{7}$ The focus is now changing. ${ }^{8}$ A recent review by the World Health Organization found that low cost simple and acceptable interventions in households can improve the biological quality of water stored in the home and hence reduce the risk of diarrhoea and death. ${ }^{2}$ Such interventions include boiling, chlorination, and coagulation-flocculation. Unfortunately, boiling consumes a lot of energy (it takes $1 \mathrm{~kg}$ of wood to boil 1 litre of water) and the cost may be prohibitive, particularly in the developing countries where wood and other biomass fuels are not always available. Moreover, burning wood can lead to deforestation with serious environmental degradation. ${ }^{2}$

Various interventions may remove particles and microbes from water. Although cloth has been found to remove zooplankton and phytoplankton carrying Vibrio cholerae $e^{9}$ and is used extensively for the eradication of guinea worm, cloth is not recommended for routine treatment of water in the home because its pores are too large to remove bacteria and viruses. Chemical precipitation (coagulation and flocculation) removes particles and microbes. It can be used in households to reduce transmission of diarrhoeal disease but its use in developing countries has been limited by issues of safety, effectiveness, cost, and sustainability. ${ }^{10}$

This is why the paper by Crump et al in this week's $B M J$ is an important advance in treating water in households. ${ }^{11}$ In a randomised controlled trial, the authors compared standard practice with flocculant-disinfectant treatment of drinking water with sodium hypochlorite (bleach) in homes in a rural area of western Kenya, where the water is highly turbid and contaminated with faecal bacteria. The treatment lowered the turbidity of drinking water, improved the acceptability of water treatment at home, and reduced the prevalence of diarrhoea by $25 \%$ among participants.

The authors also report fewer deaths in the intervention group than in the control group. It is not clear, however, whether the study had enough power to detect a significant difference in mortality, and these results on mortality reduction need to be confirmed in an appropriately powered, randomised controlled trial. The authors claim that, if the flocculant-disinfectant treatment were available in the marketplace, the visible effect on water turbidity might lead families to treat water in their homes. Yet there is no evidence to show that, in this community or indeed in other communities in the region, people would be encouraged by such results to purchase.

This study is, nevertheless, an important addition to the list of randomised controlled trials on the effect of flocculant-disinfectant on the quality and acceptability of drinking water. The challenge now is to demonstrate affordability, sustainability, and the feasibility of scaling up such interventions to reach the millions of households in developing countries that lack safe drinking water.

James K Tumwine professor

Department of Paediatrics and Child Health, Makerere Medical School, PO Box 7072, Kampala, Uganda (jtumwine@imul.com)

Competing interests: None declared.

1 Kosek M, Bern C, Guerrant RL. The global burden of diarrhoea disease, as estimated from studies published between 1992 and 2000. Bull WHO 2003;81:197-204

2 Sobsey DM. Managing water in the home: accelerated health gains from improved water supply. Geneva: World Health Organization, 2005. www.who.int/water_sanitation-health/dwq/wsh0207/en (accessed 16 Aug 2005).

3 Knight SM, Toodayan W, Caique WC, Kyin W, Barnesa A, Desmachelier P. Risk factors for the transmission of diarrhoea in children: a case control study in Malaysia. Int J Epidemiol 1992;21:812-8.

4 Quick RE, Kimura A, Thevos A, Tembo M, Shamputa I, Hutwagner L, et Quick RE, Kimura A, Thevos A, Tembo M, Shamputa I, Hutwagner L, et
al. Diarrhoea prevention through household-level water disinfection and al. Diarrhoea prevention through household-level water d
safe storage in Zambia. Am J Trop Med Hyg 2002;66:584-9.

safe storage in Zambia. Am J Trop Med Hyg 2002;66:584-9.
Mintz ED, Reiff FM, Tauxe RV. Safe water treatment and storage in the Mintz ED, Reiff FM, Tauxe RV. Safe water treatment and storage in the
home: a practical new strategy to prevent water-borne disease. JAMA 1995;273:948-53.

6 Meinhardt PL, Casemore DP, Miller KB. Epidemiological aspects of human cryptosporidiosis and the role of waterborne transmission. Epidemiol Rev 1996;18:118-36.

7 Esrey SA, Habicht J-P. Epidemiologic evidence for health benefits from improved water and sanitation in developing countries. Epidemiol Rev 1986;8:117-28.

8 Clasen TF, Cairncross S. Household water management: refining the dominant paradigm. Trop Med Int Health 2004:9:187-91.

9 Huq A, Xu B. A simple filtration method to remove plankton-associated Huq A, Xu B. A simple filtration method to remove plankton-associated
Vibrio cholerae in raw water supplies in developing countries. Appl Envi-
ron Microbiol 1996:62.2508-12. ron Microbiol 1996;62:2508-12.

10 Reller ME, Mendoza CE, Lopez BM, Alvarez M, Hoekstar RM. A randomized controlled trial of household-based flocculant-disinfectant drinking water treatment for diarrhoea prevention in rural Guatemala. Am J Trop Med Hyg 2003;69:411-19.

11 Crump JA, Otieno PO, Slutsker L, Keswick BH, Rosen DH, Hoekstar RM, et al. A cluster randomised trial of household-based flocculantdisinfectant drinking water treatment for diarrhoea prevention in the ding of highly turbid source water in rural western Kenya. BMJ 2005;331: ting of highly turbid source water in rural western Kenya. BMJ 2005;331:

\title{
Avoiding rabies
}

\section{Get vaccinated before travel, avoid animals, and get help urgently if bitten}

$\overline{\text { Clinical review } \mathrm{p} 501}$ The death from rabies of a British woman, bitten by a dog in Goa, reported in the news ${ }^{1}$ and in an article in this week's $B M J{ }^{2}$ highlights the issue of rabies prophylaxis for people who travel to and live in endemic areas. Rabies is an acute, incurable, viral encephalomyelitis caused by a bullet shaped RNA transmissible to humans. Worldwide, most of the 30000 to 70000 human deaths annually result from dog bites, although cats and wild animals such as foxes, jackals, wolves, mongooses, racoons, skunks, and bats are the other culprits. ${ }^{4}$

Around $90 \%$ of deaths from rabies occur in the developing world, with more than half in the Indian 
subcontinent, where dogs that roam freely are largely responsible. The virus is transmitted through a transdermal bite or scratch, or by contamination with animal saliva of oral mucosa or skin wounds. The incubation period is variable but is typically from one to three months, ${ }^{4}$ after which clinical symptoms appear and a fatal outcome is almost inevitable. Since rabies cannot be treated, prevention and prophylaxis are paramount.

Vaccination before exposure does not eliminate the need for treatment after rabies exposure ${ }^{5-8}$ but it simplifies treatment and may provide protection after unrecognised exposure. Currently available rabies vaccines containing inactivated virus derived through tissue culture are safe and effective. For prophylaxis before exposure, three doses of vaccine are given into the deltoid muscle on days 0,3 , and 28 , and boosters can be given between six and 24 months. Prophylaxis after exposure aims to neutralise the virus before it can enter the nervous system and entails wound cleaning, passive immunisation with immunoglobulin, and active immunisation with vaccine.$^{5-8}$ People who have completed a course of pre-exposure vaccination do not require immunoglobulin and need fewer doses of vaccine in their prophylaxis regimen after exposure: such treatment is effective in preventing the development of rabies if the recommended regimen is followed.

Wound care is essential to prevent rabies infection. The wound needs to be thoroughly scrubbed with soap and water, or, if available, iodine solution, $40-70 \%$ alcohol, cetrimide $0.1 \%$ or a similar compound, or the virucidal agent povidone, all under local anaesthesia if possible. The rabies virus is killed by sunlight, drying, soap, and the other agents mentioned. In animal experiments, early effective wound cleaning has been shown to prevent rabies infection. ${ }^{9}$

Immunisation is a medical urgency after wound cleaning, although not a medical emergency. ${ }^{5-8}$ It should be administered within 24 hours of exposure if at all possible. Rabies immunoglobulin provides rapid, passive immunity for a short time in a previously unvaccinated patient until vaccination has had time to prompt an active immune response. Immunoglobulin should be given as a single dose and infiltrated around the wound if feasible. In the developed world, human rabies immunoglobulin is the standard, but it is very expensive and not commonly available in developing countries, where equine rabies immunoglobulin may be used instead. ${ }^{7}$ Equine immunoglobulin may, however, cause an allergic reaction. A previously vaccinated patient does not require immunoglobulin and so does not face the difficulty of obtaining it in a developing country.

People who have not been vaccinated before should have post-exposure vaccination of four to five doses over four weeks, but those previously vaccinated can have boosters only on days 0 and $3 .^{5-8}$ In India, cheaper rabies vaccines derived from sheep brains are sometimes still used instead of the safer cell culture vaccines. ${ }^{10}$ Sheep brain vaccines carry a risk of serious adverse effects, such as neuritis and encephalitis. The cost of both pre-exposure and post-exposure vaccination with cell culture vaccines can be more than halved by giving a lower dose intradermally, rather than intramuscularly, but this requires greater technical skill. ${ }^{11}$

Sadly, those at greatest risk of contracting rabies from dog bites are children living in regions of the developing world where rabies is endemic. ${ }^{12}$ For travellers to those regions, particularly to remote rural areas, making a decision on pre-exposure rabies vaccination requires an assessment of the risk of being bitten and needing treatment, ${ }^{13}$ and of local access to safe and effective rabies immunoglobulin and vaccines. In many countries of Africa, Asia, Latin America, and the Middle East, dog rabies is common.

The probability of contact with an infected animal depends in part on anticipated activities, locality, and length of time spent in the country. For example, hiking in a rural area of the Indian subcontinent where dogs commonly roam free carries a sufficient risk of exposure-combined with potential difficulties in obtaining early, safe, and effective post-exposure prophylaxis-to warrant vaccination before travelling. ${ }^{5}$ The risk can be reduced by avoiding contact with animals that might be susceptible to rabies. Ignoring freely roaming dogs and cats may go against the instincts of animal loving travellers to developing countries but is a necessary precaution. Wildlife should be appreciated at a distance.

Derrick Pounder professor of forensic medicine

Department of Forensic Medicine, University of Dundee, Dundee DD1 4HN

(d.jpounder@dundee.ac.uk)

Competing interests: None declared.

1 Mudur G. Foreign visitors to India are unaware of rabies risk. $B M J$ 2005;331:255.

2 Solomon T, Marston D, Mallewa M, Felton T, Shaw S, McElhinney L, et al. Paralytic rabies after a two week holiday in India. BMJ 2005;331:501-3.

3 Pounder DJ. Rabies, lyssaviruses and bats. Scot Med J 2003;48:99-101.

4 Rupprecht CE, Hanlon CA, Hemachudha T. Rabies re-examined. Lancet 2002;2:101-9.

5 Hankins DG, Rosekrans JA. Overview, prevention and treatment of rabies. Mayo Clin Proc 2004;79:671-6.

6 McKay N, Wallis I. Rabies: a review of UK management. Emerg Med J 2005;22:316-21.

7 Ali NS. Guidelines for prophylaxis of rabies in Pakistan. Pak J Med Sci 2003; 19:61-5.

8 Rose VL. CDC issues revised guidelines for the prevention of human Rose VL. CDC issues revised guidelines for
rabies. Am Family Physician 1999;59:2007-13.

9 Dean D, Baer G, Thowpson W. Studies on the local treatment of rabies infected wounds. Bull WHO 1963;28:477.

10 Bagchi S. Preventable rabies deaths because of wrong vaccine. Lancet Neurol 2004;3:639.

11 Dutta Jk, Dutta TK. Rabies in endemic countries. BMJ 1994;308:488-9.

12 World Health Organization. Human and animal rabies. www.who.int/ rabies/en/ (accessed 16 Aug 2005).

13 Meslin F-X. Rabies as a traveller's risk, especially in high-endemicity areas. J Travel Med 2005;12(suppl):30-40. 